\title{
A Parameter-free Label Propagation Algorithm for Person Identification in Stereo Videos
}

\author{
Chongsheng Zhang ${ }^{\mathrm{a}}$, Jingjun $\mathrm{Bi}^{\mathrm{a}}$, Changchang $\mathrm{Liu}^{\mathrm{a}}$, Ke Chen ${ }^{\mathrm{b}, *}$ \\ ${ }^{a}$ School of Computer Science, Henan University, Kaifeng 475001, P.R. China \\ ${ }^{b}$ Department of Signal Processing, Tampere University of Technology, Tampere 33720, Finland
}

\begin{abstract}
Motivated by relaxing expensive and laborious person identity annotation in stereo videos, a number of research efforts have recently been dedicated to label propagation. In this work, we propose two heuristic label propagation algorithms for annotating person identities in stereo videos under the observation that the actors in two consecutive facial images in a video are more likely to be identical. In the light of this, after adjacent video frames divided into several groups, we propose our first algorithm (i.e. ZBLC4) to automatically annotate the unlabeled images with the one having the maximum summed similarity between unlabeled and labeled images in each group in the parameter-free manner. Moreover, to cope with singleton groups, an additional classifier is introduced into ZBLC4 algorithm to mitigate the suffering of unreliable prediction dependent on neighbours. We conduct experiments on three publicly-benchmarking stereo videos, demonstrating that our algorithms are superior to the state-of-the-arts.

Keywords: Label Propagation, Stereo videos, Parameter-free, Semi-supervised
\end{abstract}

${ }^{*}$ Tel.: +358-466459305; Fax.: +358-33641352.

Email address: ke. chen@tut. fi (Ke Chen) Preprint submitted to Neurocomputing

March 17, 2016 


\section{Introduction}

Automatic image annotation (labeling) is aimed to relax the requirement of expensive and laborious data annotation. However, such a problem is very challenging to annotate the persons (actors) appearing in a video due to the changes of lighting conditions and viewing angles. To solve this problem, recent research efforts have focused on label propagation [1], which is a semi-automatic process; i.e., semantic labels from a small number of manually-annotated data can be spread to a large number of unlabeled data. Most label propagation methods are designed based on similarity graphs, which infer the labels of the unlabeled images via graph travelling from labeled nodes to unlabeled ones. Considering visual information usually from two channels in stereo videos [2, 3], the information from all similarity graphs is utilized in the label propagation methods.

The accuracies of label propagation methods rely on two issues. On one hand, how to choose appropriate nodes (representation) and pair-wise similarity metrics to construct the similarity graph plays an important role. For person identification, the typical method for feature dimensionality reduction is Locality Preserving Projection (LPP) $[4,5,6,7]$. On the other hand, the accuracies of the label propagation algorithms also depend on label inference method. A number of existing works in $[8,9,10,11,12,13]$ have attempted to tackle with this problem. Our work focuses on coping with the latter.

The aim of this work is to design a parameter-free label propagation algorithm for person identification in a stereo video by discovering the temporal similarity across facial images in the consecutive frames. In our framework, we first extract facial foreground and select one facial image for each shot, which can be assumed to have the identical person in all frames. Pariwise similarities between the representative facial images are measured, which are then converted into a 
similarity matrix. With such a similarity matrix in a sequential order of videos, we can investigate a number of label propagation algorithms for automatic person identification in a video.

We propose a heuristic but effective label propagation algorithm, namely ZBLC4, which considers the sequential characteristic of the representative images of each video shot, under the assumption that the actors in two consecutive facial images are more likely to be identical. It first separates the adjacent and similar images into different groups, and then chooses the label having the maximum summed similarity between each of the unlabeled images in the group and any of the labeled images for each group. For the singleton groups from ZBLC4 that have only one image, we devise a revised ZBLC4 algorithm, namely ZBLC5, that makes use of a classification model (e.g., Support Vector Machines) trained from the labeled images to infer the labels of the images in the singleton groups. We test our proposals on three stereo films adopted in the experiments of [3]. Results show that ZBLC4 and ZBLC5 have better classification accuracies than the state-of-the-art algorithms. Moreover, the prediction accuracies increase significantly by $22 \% \sim 36 \%$ over SVM based on the libSVM toolbox.

The remaining of this paper is organized as follows. In Section 2, we investigate the related work on label propagation. Section 3 presents problem formulation. In Section 4, we propose the ZBLC4 and ZBLC5 algorithms for label propagation as well as other baseline methods. We report the results of different algorithms in Section 5. Finally, conclusion is given in Section 6.

\section{Related Work}

In recent years, a great many research efforts have been made on label inference. Label inference on the unlabeled data was designed in an iterative manner 
to converge to a stationary state $[10,14]$. A random-walk method was employed in $[15,16]$ to compute the travelling random steps in the similarity graph from the labeled nodes to the unlabeled ones The works in $[10,17,18]$ defined and utilized a smooth classification function on the graph nodes by minimizing a regularized object function that penalizes the divergence of the output labels from the initial available labels.

Label propagation algorithms in $[18,19,20,21,22]$ can share the same heuristic theory named Cluster Assumption which includes local consistence among neightboring points and global consistence within a cluster or a manifold. Orthodox supervised learning algorithms, such as k-Nearest Neighbors, are in general exploited based on the first assumption. In [10], by adopting such a heuristic assumption, their framework can spread the label information of each point to its neighbors in an iterative manner until its convergence to a global stable state. Our proposed ZBLC4 and ZBLC5 algorithms are designed under the same assumption. A general regularization framework on directed graphs was proposed in [23], which can be applied directly to bipartite graphs. Different from undirected (weighted) graphs [10, 22, 24, 25, 26], such a framework assumed the edge between two nodes indicating the class-specific likelihood of the nodes.

For person identification in a video, the labels for person identities can be spread by performing the Linear Neighborhood Propagation (LNP) method [27]. Recently, Zoidi et al. [2] extended LNP method by incorporating the visual information from the two channels of a stereo video. Our direct competitor MLPP-CLP [3] was proposed by propagating person identity labels to other unannotated facial foreground generated from video frames of stereo films, which clustered the representative facial detected foreground of each video shot and 
calculated pairwise similarities to generate a similarity matrix. Based on this similarity matrix, the theory presented in [10] is exploited for label propagation in MLPP-CLP [3]. In this paper, instead of using [10], we will develop two heuristic label propagation algorithms (ZBLC4 and ZBLC5) for person identity annotation in stereo videos.

\section{Problem Formulation}

\subsection{Problem Definition}

Given a stereo video, a video shot detection algorithm is applied to generate $N$ sequential shot cuts, with each shot cut having several facial images of the same actor/actress. After shot boundary detection, we first apply a face detection and tracking algorithm to generate a set of pairs of facial images from two video channels (i.e. left and right video channels). For simplification, we only keep the facial images detected in both channels, which are then tracked until the video shot boundary. We then select one representative facial image for each shot cut, which has the highest similarity to all others in the shot. Let $\mathscr{A}$ be the set of all the representative facial images in the video. Since there are $N$ shot cuts, the size of $\mathscr{A}$ is $N$. Let the total number of actors in the video be $C$; For exploiting stereo information, we construct a weight matrix for each channel to estimate pairwise similarity across facial images in each channel and then combine both weight matrices as [2,3] to obtain $\boldsymbol{W} \in \mathbb{R}^{N \times N}$ be a symmetric matrix that contains the similarities between the representation of paired facial images. For example, $\boldsymbol{W}(i, j)$ denotes the similarity between the $i$ th and $j$ th facial images in $\mathscr{A}$. The larger the value of $W(i, j)$ is, the more similarity two images share. Note that, different shot cuts in the stereo videos may share the same actor/actress. 
Let $\mathscr{B}$ be the subset of labeled facial images in $\mathscr{A}$, i.e., the actor/actress in each facial image in $\mathscr{B}$ is given, while the label (i.e., actor/actress) for each facial image in the set $\{\mathscr{A}-\mathscr{B}\}$ is unknown. The size of $\{\mathscr{A}-\mathscr{B}\}$, i.e., the set of facial images without labels, is $N-E$, where $E$ is the size of labeled set $\mathscr{B}$. Note that, the size of unlabeled data $N-E$ is generally much larger than that of labeled data $E$.

The problem of semi-automatic person identity annotation to be addressed in this paper is: given $\mathscr{B}$ and $W$, how can we more accurately propagate the labels of the facial images in $\{\mathscr{A}-\mathscr{B}\}$ from the labels of images in $\mathscr{A}$ ?

\subsection{A Toy Example}

Table 1: A toy example having 12 facial images.

\begin{tabular}{|c|c|c|c|c|c|c|c|c|c|c|c|c|}
\hline Image & $\mathrm{I}_{1}$ & $\mathrm{I}_{2}$ & $\mathrm{I}_{3}$ & $\mathrm{I}_{4}$ & $\mathrm{I}_{5}$ & $\mathrm{I}_{6}$ & $\mathrm{I}_{7}$ & $\mathrm{I}_{8}$ & $\mathrm{I}_{9}$ & $\mathrm{I}_{10}$ & $\mathrm{I}_{11}$ & $\mathrm{I}_{12}$ \\
\hline Label & & & 2 & 3 & & & & 1 & 1 & & 3 & \\
\hline
\end{tabular}

Table 2: Similarity matrix of the toy example in Tabel 1.

$$
\boldsymbol{W}=\left[\begin{array}{cccccccccccc}
0 & 0.6 & 0 & 0 & 0 & 0 & 0.4 & 0.5 & 0 & 0 & 0.2 & 0 \\
0.6 & 0 & 0 & 0.3 & 0 & 0 & 0.2 & 0 & 0 & 0 & 0 & 0.1 \\
0 & 0 & 0 & 0 & 0.1 & 0 & 0 & 0 & 0 & 0 & 0 & 0 \\
0 & 0.3 & 0 & 0 & 0.8 & 0 & 0 & 0 & 0 & 0 & 0 & 0 \\
0 & 0 & 0.1 & 0.8 & 0 & 0 & 0 & 0 & 0 & 0 & 0 & 0 \\
0 & 0 & 0 & 0 & 0 & 0 & 0 & 0 & 0 & 0 & 0 & 0 \\
0.4 & 0.2 & 0 & 0 & 0 & 0 & 0 & 0.4 & 0 & 0 & 0 & 0 \\
0.5 & 0 & 0 & 0 & 0 & 0 & 0.4 & 0 & 0 & 0 & 0 & 0 \\
0 & 0 & 0 & 0 & 0 & 0 & 0 & 0 & 0 & 0 & 0 & 0 \\
0 & 0 & 0 & 0 & 0 & 0 & 0 & 0 & 0 & 0 & 0 & 0 \\
0.2 & 0 & 0 & 0 & 0 & 0 & 0 & 0 & 0 & 0 & 0 & 0.7 \\
0 & 0.1 & 0 & 0 & 0 & 0 & 0 & 0 & 0 & 0 & 0.7 & 0
\end{array}\right] \in \mathbb{R}^{12 \times 12}
$$

For the convenience of presentation and explanation, we consider a toy example in Table 1 having 12 representative facial images in $\mathscr{A}$. Labeled data set $\mathscr{B}$ contains $\left\{\mathrm{I}_{3}, \mathrm{I}_{4}, \mathrm{I}_{8}, \mathrm{I}_{9}, \mathrm{I}_{11}\right\}$ as well as their corresponding labels. In Table 2, $W$ represents the similarity matrix between each pair of the 12 facial images, which can be generated by choosing an appropriate similarity metric on imagery representation in experiments. There are three labels in this example, i.e., $C=3$. 


\section{Solutions}

\subsection{Nä̈ve Algorithm}

To cope with the problem, we first propose a naïve solution, which contains two steps

- Step (1): group the facial images with similarities greater than 0. For the toy example given in Section 3.2, we will have four groups, i.e., $\left\{\mathrm{I}_{1}, \mathrm{I}_{2}, \mathrm{I}_{3}\right.$, $\left.\mathrm{I}_{4}, \mathrm{I}_{5}, \mathrm{I}_{7}, \mathrm{I}_{8}, \mathrm{I}_{11}, \mathrm{I}_{12}\right\},\left\{\mathrm{I}_{6}\right\},\left\{\mathrm{I}_{9}\right\},\left\{\mathrm{I}_{10}\right\}$

- Step (2): for each group from Step (1), adopt the majority voting strategy, i.e., use the label that appears the most times in the labeled images in this group as the label for all the images in the group; in case none of the images in the group is labeled, then the group has no label.

Finally, we will have the following prediction results using the naïve algorithm, as shown in Table 3 .

Table 3: A naïve algorithm with the toy example.

\begin{tabular}{|c|c|c|c|c|c|c|c|c|c|c|c|c|}
\hline Image & $\mathrm{I}_{1}$ & $\mathrm{I}_{2}$ & $\mathrm{I}_{3}$ & $\mathrm{I}_{4}$ & $\mathrm{I}_{5}$ & $\mathrm{I}_{6}$ & $\mathrm{I}_{7}$ & $\mathrm{I}_{8}$ & $\mathrm{I}_{9}$ & $\mathrm{I}_{10}$ & $\mathrm{I}_{11}$ & $\mathrm{I}_{12}$ \\
\hline Label & 3 & 3 & 2 & 3 & 3 & & 3 & 1 & 1 & & 3 & 3 \\
\hline
\end{tabular}

\subsection{ZBLC1 Algorithm}

ZBLC1 is a heuristic algorithm for predicting the labels of the facial images in unlabeled data set $\{\mathscr{A}-\mathscr{B}\}$. The basic idea of ZBLC1 is: for each unlabeled image $\mathrm{I}_{x}$ in $\{\mathscr{A}-\mathscr{B}\}$, the label of an image (denoted as $\mathrm{I}_{y}$ ) in labeled data set $\mathscr{B}$ that has the greatest similarity with $\mathrm{I}_{x}$ will be utilized as the label of $\mathrm{I}_{x}$.

For the toy example given in section $3.2, I_{1}$ is an unlabeled image, $\left\{\mathrm{I}_{8}, \mathrm{I}_{11}\right\}$ is the set of labeled images whose similarities with $\mathrm{I}_{1}$ are greater than 0 . Since 
the similarity between $I_{8}$ and $I_{1}$ is the larger one, the label of $I_{8}$ will be annotated for $I_{1}$. Finally, using the ZBLC1 algorithm, we will obtain the prediction results shown in Table 4.

Table 4: ZBLC1 algorithm with the toy example.

\begin{tabular}{|c|c|c|c|c|c|c|c|c|c|c|c|c|}
\hline Image & $\mathrm{I}_{1}$ & $\mathrm{I}_{2}$ & $\mathrm{I}_{3}$ & $\mathrm{I}_{4}$ & $\mathrm{I}_{5}$ & $\mathrm{I}_{6}$ & $\mathrm{I}_{7}$ & $\mathrm{I}_{8}$ & $\mathrm{I}_{9}$ & $\mathrm{I}_{10}$ & $\mathrm{I}_{11}$ & $\mathrm{I}_{12}$ \\
\hline Label & 1 & 3 & 2 & 3 & 3 & & 1 & 1 & 1 & & 3 & 3 \\
\hline
\end{tabular}

\subsection{ZBLC2 Algorithm}

ZBLC2 is another heuristic algorithm that takes the sequential characteristic of the images in a video into consideration under the assumption that the persons in two consecutive facial images are more likely to be identical. Based on such an observation, ZBLC2 algorithm develops the following framework to predict the labels of the facial images in $\{\mathscr{A}-\mathscr{B}\}$.

- Step (1): for each unlabeled image $\mathrm{I}_{x}$ in $\{\mathscr{A}-\mathscr{B}\}$, if 1) at least one neighboring image of $\mathrm{I}_{x}$ is labeled; and 2) the similarity between $\mathrm{I}_{x}$ and its labeled neighbor(s) is greater than 0 , then use the label of its labeled neighbor that has the larger similarity with $\mathrm{I}_{x}$ as the label of $\mathrm{I}_{x}$. Repeat the above process until no more unlabeled images can be annotated.

- Step (2): adopt ZBLC1 algorithm to label the remaining unlabeled images in $\{\mathscr{A}-\mathscr{B}\}$.

Consider again the toy example in Section 3.2, using ZBLC2 algorithm, in Step (1), the label of $I_{5}$ will be the same as that of $I_{4}$ whose label is given. Similarly, the label of $\mathrm{I}_{12}$ will be the same as $\mathrm{I}_{11}$. As a consequence, we will have the results reported in Table 5. In Step (2), we adopt ZBLC1 algorithm to predict the rest unlabeled images. Finally, using the ZBLC2 algorithm, we will obtain the results in Table 6 . 
Table 5: Step (1) in ZBLC2 algorithm with the toy example.

\begin{tabular}{|c|c|c|c|c|c|c|c|c|c|c|c|c|}
\hline Image & $\mathrm{I}_{1}$ & $\mathrm{I}_{2}$ & $\mathrm{I}_{3}$ & $\mathrm{I}_{4}$ & $\mathrm{I}_{5}$ & $\mathrm{I}_{6}$ & $\mathrm{I}_{7}$ & $\mathrm{I}_{8}$ & $\mathrm{I}_{9}$ & $\mathrm{I}_{10}$ & $\mathrm{I}_{11}$ & $\mathrm{I}_{12}$ \\
\hline Label & & & 2 & 3 & 3 & & 1 & 1 & 1 & & 3 & 3 \\
\hline
\end{tabular}

Table 6: Step (2) in ZBLC2 algorithm with the toy example.

\begin{tabular}{|c|c|c|c|c|c|c|c|c|c|c|c|c|}
\hline Image & $\mathrm{I}_{1}$ & $\mathrm{I}_{2}$ & $\mathrm{I}_{3}$ & $\mathrm{I}_{4}$ & $\mathrm{I}_{5}$ & $\mathrm{I}_{6}$ & $\mathrm{I}_{7}$ & $\mathrm{I}_{8}$ & $\mathrm{I}_{9}$ & $\mathrm{I}_{10}$ & $\mathrm{I}_{11}$ & $\mathrm{I}_{12}$ \\
\hline Label & 1 & 3 & 2 & 3 & 3 & & 1 & 1 & 1 & & 3 & 3 \\
\hline
\end{tabular}

\subsection{ZBLC3 Algorithm}

Based on ZBLC2, we develop the ZBLC3 algorithm, Step (1) of which is the same as that of ZBLC2. However, in Step (2) of ZBLC3, instead of using ZBLC1, we adopt a strategy that has two sub-steps that are very similar to two steps of the naïve algorithm in Section 4.1. The only difference is that, in the first sub-step in Step (2), for further processing the results after Step (1), we add another constraint when grouping the unlabeled facial images with similarities greater than 0 . That is, only the adjacent and similar images can be put in the same group. Consequently, there will be more groups in the first sub-step of Step (2) in ZBLC3 than Step (1) of the naïve algorithm. Considering again the toy example, we will obtain the following results using ZBLC3, which is shown in Table 7.

Table 7: ZBLC3 algorithm with the toy example.

\begin{tabular}{|c|c|c|c|c|c|c|c|c|c|c|c|c|}
\hline Image & $\mathrm{I}_{1}$ & $\mathrm{I}_{2}$ & $\mathrm{I}_{3}$ & $\mathrm{I}_{4}$ & $\mathrm{I}_{5}$ & $\mathrm{I}_{6}$ & $\mathrm{I}_{7}$ & $\mathrm{I}_{8}$ & $\mathrm{I}_{9}$ & $\mathrm{I}_{10}$ & $\mathrm{I}_{11}$ & $\mathrm{I}_{12}$ \\
\hline Label & 1 & 1 & 2 & 3 & 3 & & 1 & 1 & 1 & & 3 & 3 \\
\hline
\end{tabular}

\subsection{ZBLC4 Algorithm}

With other parts of ZBLC3 remaining the same in ZBLC4, we further propose the ZBLC4 algorithm that replaces the majority voting in the second substep of Step (2) in ZBLC3 by another strategy. Such a strategy chooses for each group, the label that has the maximum summed similarity between each of the 
unlabeled images in the same group and any of the labeled images from Step (1). The overall pseudo code of the algorithm is presented in Algorithm 1.

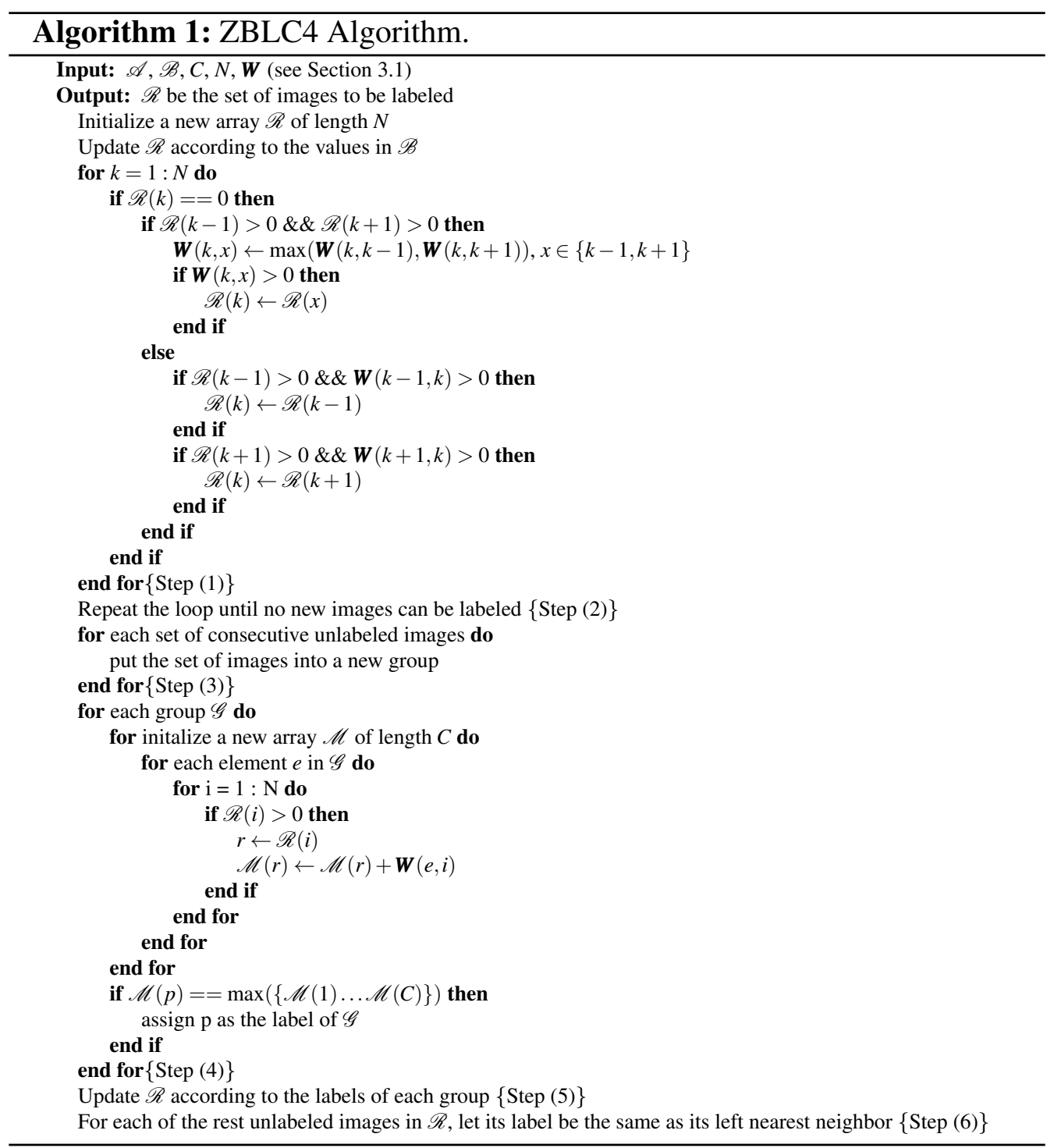

Simply put, Step (1) of Algorithm 1 in ZBLC4 is the same as that in ZBLC3 and ZBLC2, and then the remaining consecutive unlabeled images are grouped and will be annotated with the same labels. Finally, the summed similarity be- 


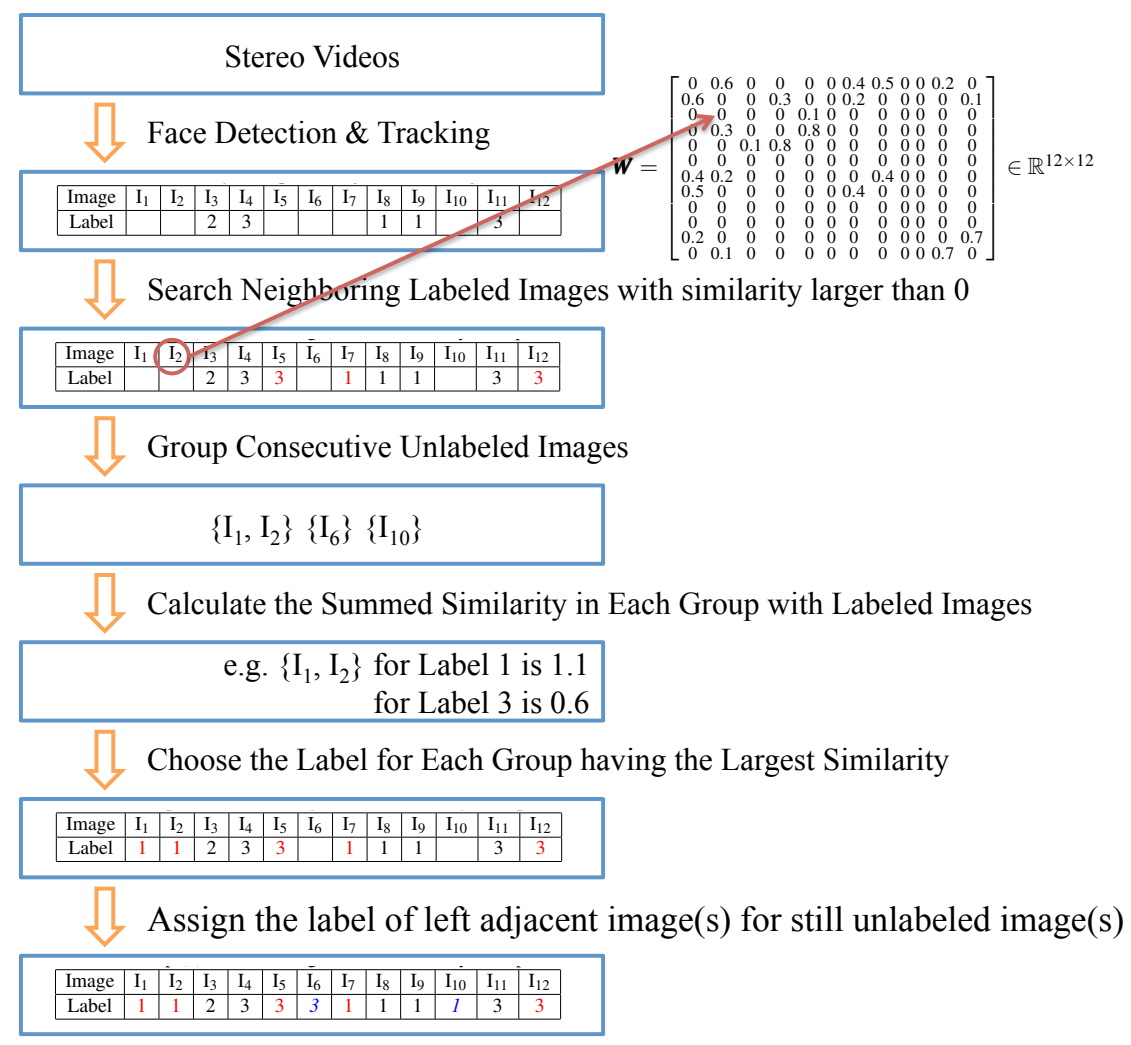

Figure 1: The pipeline of ZBLC4 with a toy example.

tween each element in the unlabeled groups and labeled images belonging to the same label group will be calculated and the label having the maximum similarity will be annotated for this unlabeled group. For illustrative purpose, let us consider again the toy example given in Section 3.2 and the workflow of ZBLC4 is given in Figure 1. After Step (1) of ZBLC4, we shall obtain the same result as the result of the Step (1) in ZBLC3. In the first sub-step of Step (2) in ZBLC4, we will have three groups $\left\{\mathrm{I}_{1}, \mathrm{I}_{2}\right\},\left\{\mathrm{I}_{6}\right\}$ and $\left\{\mathrm{I}_{10}\right\}$. To determine the label for the group $\left\{\mathrm{I}_{1}, \mathrm{I}_{2}\right\}$, we find out that $\mathrm{I}_{1}$ has non-zero similarities with $\mathrm{I}_{7}, \mathrm{I}_{8}, \mathrm{I}_{11}$, while $\mathrm{I}_{2}$ has non-zero similarities with $\mathrm{I}_{4}, \mathrm{I}_{7}, \mathrm{I}_{12}$. Specifically, $\boldsymbol{W}\left(\mathrm{I}_{1}, \mathrm{I}_{7}\right)=0.4, \boldsymbol{W}\left(\mathrm{I}_{1}, \mathrm{I}_{8}\right)=0.5, \boldsymbol{W}\left(\mathrm{I}_{1}, \mathrm{I}_{11}\right)=0.2 ; \boldsymbol{W}\left(\mathrm{I}_{2}, \mathrm{I}_{4}\right)=0.3, \boldsymbol{W}\left(\mathrm{I}_{2}, \mathrm{I}_{7}\right)=$ 11 
$0.2, W\left(I_{2}, I_{12}\right)=0.1$. The label of $I_{4}$ is 3 , while the labels of $I_{11}$ and $I_{12}$ are also 3. Hence, the summed similarities for label 3 in the group $\left\{I_{1}, I_{2}\right\}$ is equal to $0.2+0.3+0.1=0.6$. Similarly, we can calculate the summed similarities for label 1 whose sum is equal to $0.4+0.5+0.2=1.1$. As the summed similarities for label 1 is the greatest value, the label of the group $\left\{I_{1}, I_{2}\right\}$ will be annotated as 1. The detailed algorithm for this step is depicted in Step (4) of Algorithm 1. After Step (4), we shall have the following results reported in Table 8. In the Table 8: Step (4) in ZBLC4 algorithm with the toy example.

\begin{tabular}{|c|c|c|c|c|c|c|c|c|c|c|c|c|}
\hline Image & $\mathrm{I}_{1}$ & $\mathrm{I}_{2}$ & $\mathrm{I}_{3}$ & $\mathrm{I}_{4}$ & $\mathrm{I}_{5}$ & $\mathrm{I}_{6}$ & $\mathrm{I}_{7}$ & $\mathrm{I}_{8}$ & $\mathrm{I}_{9}$ & $\mathrm{I}_{10}$ & $\mathrm{I}_{11}$ & $\mathrm{I}_{12}$ \\
\hline Label & 1 & 1 & 2 & 3 & 3 & & 1 & 1 & 1 & & 3 & 3 \\
\hline
\end{tabular}

Step (6) of ZBLC4, each of the rest unlabeled images will be assigned a label the same as its left adjacent neighbor. Finally, we have the following results shown in Table 9.

Table 9: Step (6) in ZBLC4 algorithm with the toy example.

\begin{tabular}{|c|c|c|c|c|c|c|c|c|c|c|c|c|}
\hline Image & $\mathrm{I}_{1}$ & $\mathrm{I}_{2}$ & $\mathrm{I}_{3}$ & $\mathrm{I}_{4}$ & $\mathrm{I}_{5}$ & $\mathrm{I}_{6}$ & $\mathrm{I}_{7}$ & $\mathrm{I}_{8}$ & $\mathrm{I}_{9}$ & $\mathrm{I}_{10}$ & $\mathrm{I}_{11}$ & $\mathrm{I}_{12}$ \\
\hline Label & 1 & 1 & 2 & 3 & 3 & 3 & 1 & 1 & 1 & 1 & 3 & 3 \\
\hline
\end{tabular}

Remarks: Compared to the aforementioned Naïve and ZBLC algorithms, ZBLC4 has the following advantages:

- Compared to Naïve and ZBLC3, ZBLC4 adopts the strategy by choosing the largest summed similarity between each element of unlabeled image groups and labeled images instead of majority voting, which is less robust against noises.

- The concept employed in ZBLC4 is very simple but effective: adjacent 
unlabeled images are more likely to share the same labels, which increases the joint similarity of those images in the same unlabeled image groups.

\subsection{ZBLC5 Algorithm}

Based on ZBLC4, we propose the ZBLC5 algorithm which combines ZBLC4 with an additional classification step for singleton groups (i.e., only one unlabeled image in each group). ZBLC5 first applies ZBLC4 to annotate the unlabeled images; but for each singleton group in the second sub-step in Step (2) of ZBLC4, we employ a classification model (typically Support Vector Machines) trained from the images in Step (1) of ZBLC4 to predict their labels for the unlabeled images in the singleton groups. Specifically, the similarity values between labeled images and their corresponding labels are fed into the multi-class classifier as the input feature and labels to train a model. During testing, the unlabeled images of singleton groups is applied to the trained model to predict their labels. For the toy example in Section 3.2, we shall have the following result with ZBLC5, where $S_{3}$ and $S_{4}$ represent the predicted results from Support Vector Classification on the singleton groups $\left\{\mathrm{I}_{6}\right\}$ and $\left\{\mathrm{I}_{10}\right\}$, respectively. The rational in ZBLC5 is: if a group contains only one image, then the prediction of ZBLC4 depending on the neighbors is more likely to be unreliable, so we count on a supervised classification model to improve the prediction performance. The results by ZBLC5 are given in Table 10 .

Table 10: ZBLC5 algorithm with the toy example.

\begin{tabular}{|c|c|c|c|c|c|c|c|c|c|c|c|c|}
\hline Image & $\mathrm{I}_{1}$ & $\mathrm{I}_{2}$ & $\mathrm{I}_{3}$ & $\mathrm{I}_{4}$ & $\mathrm{I}_{5}$ & $\mathrm{I}_{6}$ & $\mathrm{I}_{7}$ & $\mathrm{I}_{8}$ & $\mathrm{I}_{9}$ & $\mathrm{I}_{10}$ & $\mathrm{I}_{11}$ & $\mathrm{I}_{12}$ \\
\hline Label & 1 & 1 & 2 & 3 & 3 & $S_{3}$ & 1 & 1 & 1 & $S_{4}$ & 3 & 3 \\
\hline
\end{tabular}


Table 11: Details of three stereo videos. $N$ : the size of representative images in each video; $E$ : the size of labeled images in each video data; $N-E$ : the size of unlabeled images in each video data; $C$ : the number of actors; $T$ : the number of facialimage trajectories.

\begin{tabular}{|l|c|c|c|c|c|}
\hline & $N$ & $E$ & $N-E$ & $C$ & $T$ \\
\hline HUGUO & 5398 & 269 & 5129 & 27 & 1532 \\
\hline DA & 3498 & 174 & 3324 & 41 & 1435 \\
\hline POTCOST & 4954 & 247 & 4707 & 59 & 1878 \\
\hline
\end{tabular}

\section{Experiments}

Datasets and Settings - We conducted experiments on three films used in [1, 3]: HUGUO, DA and POTCOST. Each video data is extracted from a highresolution stereo-film $(1080 \times 1920$ pixels per frame). The facial foreground were detected by the revised Viola-Jones face detector [28] to incorporate color information [29] ${ }^{1}$. In view of temporal correlated between video frames, the detected facial image was tracked for the next 20 frames or until a shot boundary by a single channel appearance-based object tracker [30]. As a result, we will have a number of facial image trajectories consisting of detected foreground in stereo videos. It is assumed that facial images in the same trajectory share the same identity label, which can be represented by one image for label annotation. For available label annotation, considering the false detection, we add an additional actor class for each video, which leads to HUGUO, DA and POTCOST having 28, 42 and 60 actor classes respectively. After facial images alignment with the funnel algorithm $[31,32]$, the constructed data for semi-automatic label annotation is thus generated. More details of three videos are given in Table 11. For additional classification step for singleton groups in ZBLC5, we employed widely-used libSVM [33] in the experiments. In details, we utilise sigmoid kernel with the optimised parameters (trade-off between regularised term and loss

\footnotetext{
${ }^{1}$ The data will be released after acceptance for public evaluation as the_data_can_ downloaded_from_here. 
term $C=2$, coefficients for sigmoid kernel are $-g 1.1$ and $-r 1$ respectively) via 10-fold cross-validation. For performance metric, we evaluate all the results with classification accuracy.

Table 12: Comparison with state-of-the-art algorithms evaluated by classification accuarcy.

\begin{tabular}{|l|c|c|c|c|c|c|c|c|}
\hline & LPP [4] & OLPP [34] & PCLPP [35] & GoLPP [5] & NPE [36] & MLPP-CLP [3] & ZBLC4 & ZBLC5 \\
\hline HUGUO & $74.26 \%$ & $68.40 \%$ & $75.71 \%$ & $69.26 \%$ & $75.64 \%$ & $80.52 \%$ & $83.24 \%$ & $\mathbf{8 3 . 4 6 \%}$ \\
\hline DA & $56.05 \%$ & $48.68 \%$ & $59.77 \%$ & $59.52 \%$ & $57.46 \%$ & $67.10 \%$ & $70.90 \%$ & $\mathbf{7 1 . 3 0 \%}$ \\
\hline POTCOST & $61.50 \%$ & $63.06 \%$ & $62.54 \%$ & $58.91 \%$ & $63.14 \%$ & $68.39 \%$ & $\mathbf{7 4 . 0 8 \%}$ & $72.95 \%$ \\
\hline
\end{tabular}

Comparison with State-Of-The-Arts - We compare our ZBLC methods with state-of-the-art algorithms and the results are shown in Table 12. It is evident that the proposed ZBLC4 and ZBLC5 algorithms can significantly outperform the recently-proposed frameworks on all three video data. Using the same imagery representation because of the same processing procedure for stereo videos, the only difference between the direct competitor MLPP-CLP [3] and our methods lies in the exploitation of temporal correlation between facial images of neighboring video shot, which verify our motivation. In details, MLPP-CLP [3] adopts the ordinary clustering algorithms to generate a number of groups as the proposed ZBLC4 and ZBLC5, but we propose an additional temporal constrains to generate a number of consecutive facial image groups, which leads superior performance. Moreover, our ZBLC4 and ZBLC5 algorithms are designed in a parameter-free manner during label propagation, which could be more stable than other frameworks with the need of parameter-tuning.

Table 13: Classification accuracies of libSVM and ZBLC algorithms on three videos.

\begin{tabular}{|l|c|c|c|c|c|c|c|c|}
\hline & libSVM0 & libSVM1 & Naïve & ZBLC1 & ZBLC2 & ZBLC3 & ZBLC4 & ZBLC5 \\
\hline HUGUO & $47.42 \%$ & $69.13 \%$ & $45.92 \%$ & $67.25 \%$ & $71.80 \%$ & $81.05 \%$ & $83.24 \%$ & $\mathbf{8 3 . 4 6 \%}$ \\
\hline DA & $49.07 \%$ & $53.25 \%$ & $33.25 \%$ & $58.75 \%$ & $54.86 \%$ & $67.21 \%$ & $70.90 \%$ & $\mathbf{7 1 . 3 0 \%}$ \\
\hline POTCOST & $47.70 \%$ & $51.77 \%$ & $37.95 \%$ & $64.01 \%$ & $60.94 \%$ & $72.57 \%$ & $\mathbf{7 4 . 0 8 \%}$ & $72.95 \%$ \\
\hline
\end{tabular}




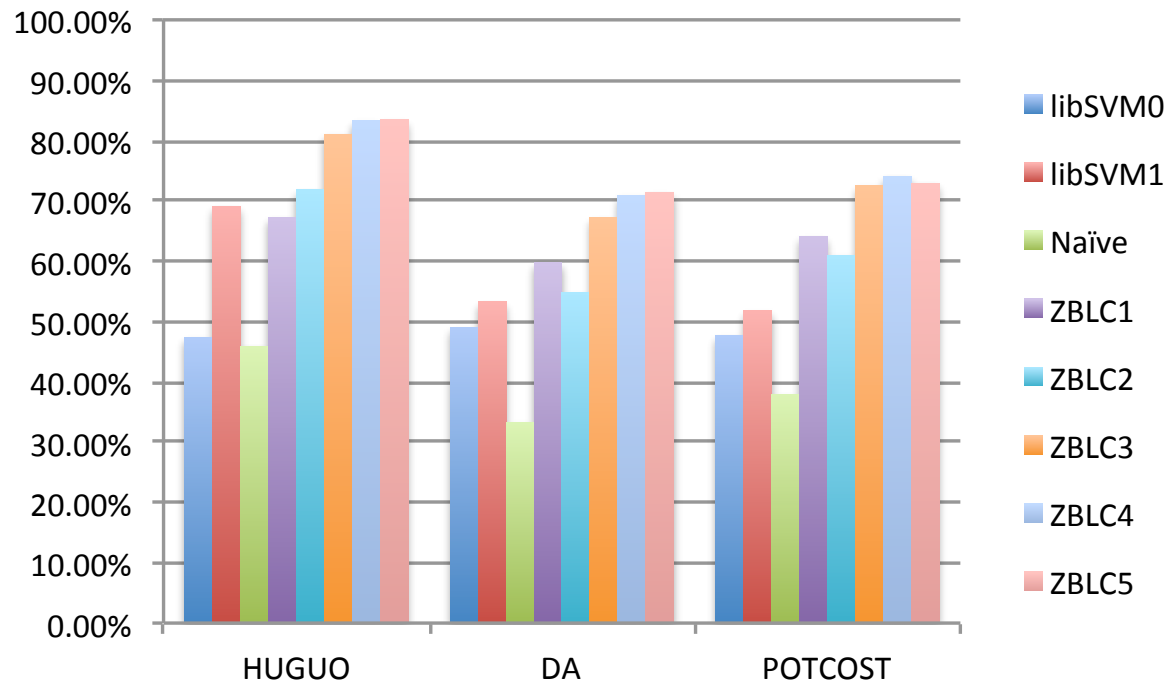

Figure 2: Classification accuracies of different algorithms on HUGUO, DA and POTCOST.

Comparison with Other ZBLC Variants - The experimental results with libSVM0, libSVM1, naïve algorithm, and ZBLC algorithms are all evaluated using the ground truth labels of each data. The accuracies of these algorithms on the HUGUO, DA and POTCOST are described in Table 13 and Figure 2. With using the libSVM library, in libSVM0, we apply Support Vector Machines (SVM) for classification directly using the available labeled data; while in libSVM1, we train a SVM model on the results obtained from the Step (1) of ZBLC4, to predict the rest unlabeled images. Evidently, ZBLC3, ZBLC4 and ZBLC5 can achieve significantly better performance than other methods. Considering the same Step (1) employed in libSVM1, ZBLC2, the only explanation for such experiment results are that the additional constraint for capturing temporal correlation between sequentially-ordered images is added, which can verify our observation. Better performance of ZBLC4 and ZBLC5 over ZBLC3 demonstrates that our strategy to choose maximum summed similarity is more favourable than majority voting. 
Table 14: Label inference from labeled neighbors on three stereo videos.

\begin{tabular}{|l|c|c|c|c|}
\hline & $N$ & $E$ & number of images in stage 1 & correct predictions in stage 1 \\
\hline HUGUO & 5398 & 269 & 2084 & 2083 \\
\hline DA & 3498 & 174 & 1175 & 1169 \\
\hline POTCOST & 4954 & 247 & 1701 & 1697 \\
\hline
\end{tabular}

Effect of Label Inference from Labeled Neighbors - The classification accuracies in the Step (1) of ZBLC4 as well as ZBLC2, ZBLC3 and ZBLC5, i.e., label inference from labeled neighbors, is as high as $99 \%$, as can be seen in Table 14. Take the HUGUO dataset as an example, initially there are in total 5398 facial images, 269 of which are labeled. At the end of Step (1) of ZBLC4, the labels of 2084 images were predicted; among them, 2083 turned out to be correct predictions. As a result, the prediction accuracies of label inference from labeled neighbors is very high. We can observe from Figure 2 that, the classification accuracies of libSVM1, ZBLC4 and ZBLC5 are much higher than libSVM0, with an increased accuracy of $22 \% \sim 36 \%$ than libSVM0, which indicates positive effect of label inference from labeled neighbors on classification performance.

Effect of Considering Trajectory Length - We now report the accuracies of all the algorithms, after considering trajectory length. Trajectory length (size: 2 by $N$ ) denotes the number of images in each video shot of the video $[1,3]$. Consequently, if the representative facial image of a shot is correctly predicted, then all the images in the shot will be correctly labeled; otherwise, all the images will be mislabeled. The results are reported in Figure 3. We can see that the ZBLC4 and ZBLC5 have the better classification performance. Compared to ZBLC4 and ZBLC5 without using trajectory length, the methods considering trajectory length suffer more from the accuracies of tracking, which are less reliable. 


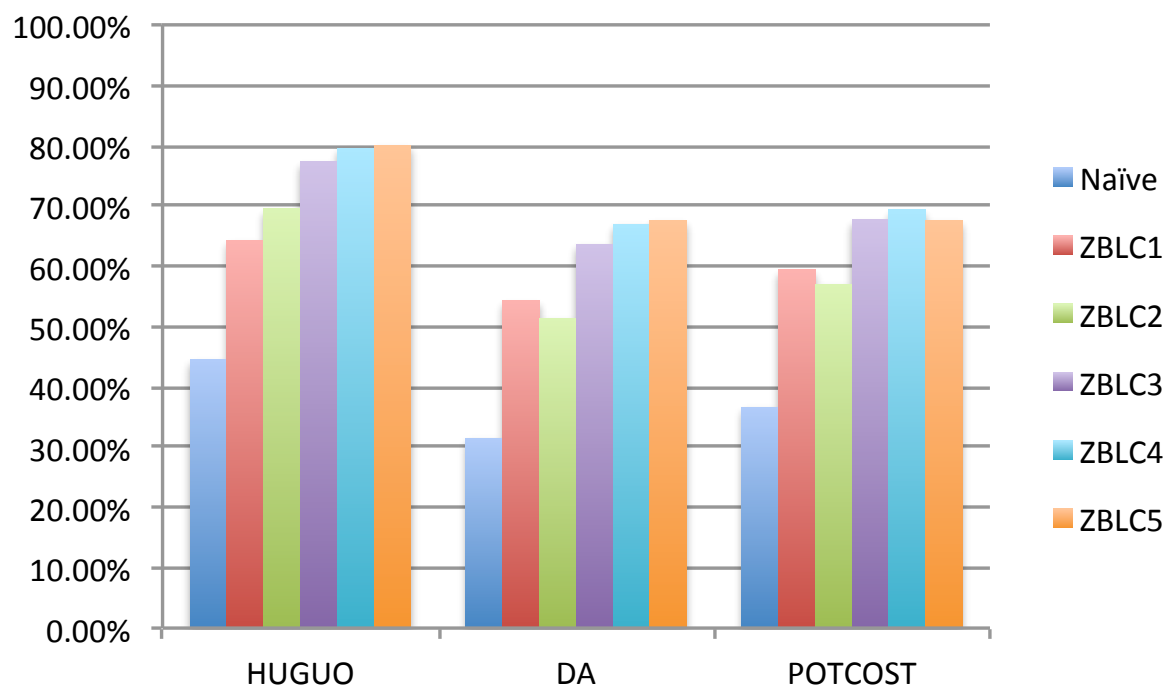

Figure 3: Classification accuracies of different algorithms on three video data when considering trajectory length.

\section{Conclusion}

In this paper, we propose a simple but effective ZBLC4 algorithm to propagate the labels of the un-annotated facial images in stereo videos using only a few available labeled data. The proposed ZBLC4 and ZBLC5 have better prediction accuracies than the state-of-the-art algorithms. Experiments on three stereo film data demonstrate that our motivation to discover and capture temporal correlation between video shots. Moreover, ZBLC4 is parameter-free: it does not require any manual effort to tune the parameters in label propagation.

\section{Acknowledgements}

This work is partially funded by the National Science Foundation of China (NSFC) with Grant number of 41401466, 61300215 and 61272545. It is also supported by Henan University under Grant number of xxjc20140005 and 2013 YBZR014. 
In addition, it is also funded by Academy of Finland under the Grant No. 267581 and D2I SHOK project funded by Digile Oy and Nokia Technologies (Tampere, Finland). The authors greatly appreciate the help from Prof. Pitas and Dr. Olga Zoidi of Aristotle University of Thessaloniki, for introducing this research problem and providing the data for experiments. The authors also acknowledge the donations of Nvidia Tesla K40 from Nvidia Corporation to the first author.

\section{References}

[1] X. Zhu, Semi-supervised learning literature survey, Tech. rep., University of Wisconsin (2008).

[2] O. Zoidi, N. Nikolaidis, I. Pitas, Exploiting clustering and stereo information in label propagation on facial images, in: IEEE Workshop on Computational Intelligence in Biometrics and Identity Management, 2013, pp. 31-35.

[3] O. Zoidi, A. Tefas, N. Nikolaidis, I. Pitas, Person identity label propagation in stereo videos, IEEE Transactions on Multimedia 16 (5) (2014) 1358-1368.

[4] X. Niyogi, Locality preserving projections, in: Neural information processing systems, Vol. 16, 2004, pp. 153-160.

[5] L. Zhang, L. Qiao, S. Chen, Graph-optimized locality preserving projections, Pattern Recognition 43 (6) (2010) 1993-2002.

[6] J. Lu, Y.-P. Tan, Regularized locality preserving projections and its extensions for face recognition, IEEE Transactions on Systems, Man, and Cybernetics, Part B: Cybernetics 40 (3) (2010) 958-963.

[7] L. Qiao, S. Chen, X. Tan, Sparsity preserving projections with applications to face recognition, Pattern Recognition 43 (1) (2010) 331-341.

[8] T. Tuytelaars, M.-F. Moens, et al., Naming people in news videos with label propagation, IEEE Multimedia 18 (3) (2011) 44-55.

[9] O. Chapelle, B. Schölkopf, A. Zien, et al., Semi-supervised learning.

[10] D. Zhou, O. Bousquet, T. N. Lal, J. Weston, B. Schölkopf, Learning with local and global consistency, Advances in neural information processing systems 16 (16) (2004) 321-328. 
[11] M. Wang, X.-S. Hua, R. Hong, J. Tang, G.-J. Qi, Y. Song, Unified video annotation via multigraph learning, IEEE Transactions on Circuits and Systems for Video Technology 19 (5) (2009) 733-746.

[12] A. Argyriou, M. Herbster, M. Pontil, Combining graph laplacians for semi-supervised learning, in: Advances in Neural Information Processing Systems, 2005, pp. 67-74.

[13] T. Kato, H. Kashima, M. Sugiyama, Robust label propagation on multiple networks, IEEE Transactions on Neural Networks 20 (1) (2009) 35-44.

[14] X. Zhu, Z. Ghahramani, Learning from labeled and unlabeled data with label propagation, Tech. rep., Carnegie Mellon University (2002).

[15] M. S. T. Jaakkola, M. Szummer, Partially labeled classification with markov random walks, Advances in neural information processing systems 14 (2002) 945-952.

[16] D. Zhou, B. Schölkopf, Learning from labeled and unlabeled data using random walks, in: Pattern Recognition, Vol. 3175, 2004, pp. 237-244.

[17] J. Tang, R. Hong, S. Yan, T.-S. Chua, G.-J. Qi, R. Jain, Image annotation by k nn-sparse graph-based label propagation over noisily tagged web images, ACM Transactions on Intelligent Systems and Technology 2 (2) (2011) 14:1-14:15.

[18] A. Blum, S. Chawla, Learning from labeled and unlabeled data using graph mincuts, in: International Conference on Machine Learning, 2001, pp. 19-26.

[19] M. Belkin, P. Niyogi, Semi-supervised learning on manifolds, Advances in Neural Information Processing Systems.

[20] O. Chapelle, J. Weston, B. Schölkopf, Cluster kernels for semi-supervised learning, in: Advances in neural information processing systems, 2002, pp. 585-592.

[21] M. Seeger, Learning with labeled and unlabeled data, Tech. rep., University of Edinburgh (2000).

[22] X. Zhu, Z. Ghahramani, J. Lafferty, et al., Semi-supervised learning using gaussian fields and harmonic functions, in: International Conference on Machine Learning, 2003, pp. 912919.

[23] D. Zhou, T. Hofmann, B. Schölkopf, Semi-supervised learning on directed graphs, in: Advances in neural information processing systems, 2004, pp. 1633-1640.

[24] M. Belkin, I. Matveeva, P. Niyogi, Regularization and semi-supervised learning on large graphs, in: Annual Conference on Learning Theory, Vol. 3120, 2004, pp. 624-638. 
[25] T. Joachims, Transductive learning via spectral graph partitioning, in: International Conference on Machine Learning, Vol. 3, 2003, pp. 290-297.

[26] A. J. Smola, R. Kondor, Kernels and regularization on graphs, in: Learning theory and kernel machines, 2003, pp. 144-158.

[27] F. Wang, C. Zhang, Label propagation through linear neighborhoods, in: International Conference on Machine Learning, 2006, pp. 985-992.

[28] P. Viola, M. Jones, Robust real-time object detection, International Journal of Computer Vision 4 (2001) 51-52.

[29] G. Stamou, M. Krinidis, N. Nikolaidis, I. Pitas, A monocular system for automatic face detection and tracking, in: Visual Communications and Image Processing, 2005.

[30] S. K. Zhou, R. Chellappa, B. Moghaddam, Visual tracking and recognition using appearance-adaptive models in particle filters, IEEE Transactions on Image Processing 13 (11) (2004) 1491-1506.

[31] L. Wolf, T. Hassner, Y. Taigman, Effective unconstrained face recognition by combining multiple descriptors and learned background statistics, IEEE Transactions on Pattern Analysis and Machine Intelligence 33 (10) (2011) 1978-1990.

[32] G. B. Huang, M. Ramesh, T. Berg, E. Learned-Miller, Labeled faces in the wild: A database for studying face recognition in unconstrained environments, Tech. rep., University of Massachusetts, Amherst (2007).

[33] C.-C. Chang, C.-J. Lin, LIBSVM: A library for support vector machines, ACM Transactions on Intelligent Systems and Technology 2 (3) (2011) 27:1-27:27.

[34] L. Zhu, S. Zhu, Face recognition based on orthogonal discriminant locality preserving projections, Neurocomputing 70 (7) (2007) 1543-1546.

[35] H. Cevikalp, J. Verbeek, F. Jurie, A. Klaser, Semi-supervised dimensionality reduction using pairwise equivalence constraints, in: International Conference on Computer Vision Theory and Applications, 2008, pp. 489-496.

[36] X. He, D. Cai, S. Yan, H.-J. Zhang, Neighborhood preserving embedding, in: IEEE International Conference on Computer Vision, 2005, pp. 1208-1213. 